\title{
Systems and Tools for Cross-Device User Interfaces
}

\author{
Michael Nebeling ${ }^{1}$, Fabio Paternò ${ }^{2}$, Frank Maurer $^{3}$, Jeffrey Nichols $^{4}$ \\ ${ }^{1}$ CMU HCII ${ }^{2}$ C.N.R.-ISTI ${ }^{3}$ Department of Computer Science, University of Calgary ${ }^{4}$ Google Inc. \\ nebeling@cmu.edu, fabio.paterno@isti.cnr.it, frank.maurer@ucalgary.ca, jeff@ jeffreynichols.com
}

\begin{abstract}
The goal of the XDUI 2015 workshop is to bring together leading and upcoming systems researchers in the area of cross-device interfaces and define a research agenda together. The workshop aims to be useful, not only for the EICS research community, but for the wider HCI community, where many recent cross-device systems and tools have been developed and investigated almost in parallel without learning from and building on each other. It targets both new and established researchers in the area-new researchers will quickly get an overview of the state of the art, while established researchers can draw more detailed comparisons between their solutions and discuss benefits and limitations. This workshop at EICS provides a unique opportunity to sketch the design space of possible cross-device user interfaces and discuss technical concerns of existing solutions as well as open issues and future research directions.
\end{abstract}

\section{Author Keywords}

multi-device, distributed user interfaces; cross-device interaction, systems and tools.

\section{MOTIVATION AND BACKGROUND}

Early notions of multi-device, distributed user interfaces were evident in the Pebbles project at Carnegie Mellon University, developing, for example, the Personal Universal Controller [17] based on a remote control metaphor for mobile devices to control various appliances. Given the recent proliferation of many new forms of devices, researchers have again started to experiment with better support for distribution of user interfaces across devices. Many different languages $[12,16,19]$, frameworks $[1,2,6,7,8,9,10,11,14$, $15,24]$ and tools $[3,4,5,13]$ have been proposed to support development of cross-device applications. In 2012, Paternò and Santoro [18] presented a thorough review of multidevice research and the different dimensions that need to be distinguished when categorising existing solutions. In addition, several user studies have been conducted to derive suitable and popular cross-device interaction techniques [20, 22, $21,23]$, but their potentail to be realised and implemented in working systems remains unexplored.

Permission to make digital or hard copies of part or all of this work for personal or classroom use is granted without fee provided that copies are not made or distributed for profit or commercial advantage and that copies bear this notice and the full citation on the first page. Copyrights for third-party components of this work must be honored. For all other uses, contact the Owner/Author.

Copyright is held by the owner/author(s).

EICS'15, June 23 - 26, 2015, Duisburg, Germany

ACM 978-1-4503-3646-8/15/06.

http://dx.doi.org/10.1145/2774225.2777463
While there has been research interest in the topic of crossdevice interfaces for many years, it is only recently that it has received increased attention with many new frameworks, platforms and toolkits being developed in research and industry. Many of the proposed solutions share similarities in terms of the approach and, to a large extent also, implementation. However, scope, portability and scalability differ significantly between the solutions making detailed comparisons a challenge. The rapid pace at which new solutions are proposed makes it both difficult to identify the contributions of individual pieces of work and maintain an overview. In particular, for new researchers in the area it is a challenge to work out the open issues. On the other hand, experienced researchers see significant overlap between recent proposals and are afraid that we are not really pushing forward as a community. Despite the body of knowledge in terms of publications, access to code and sample applications illustrating the potential and limitations of available solutions is not always given. The workshop features demos of existing solutions and allows participants to obtain an overview of the design space and identify possible future research directions. Its specific focus on systems and tools for cross-device interfaces make it a good fit to EICS and should encourage participation.

\section{ORGANISATION OF THE WORKSHOP}

This workshop brings together both new and established researchers in cross-device interfaces. To foster discussion among participants, the idea for this workshop is not to have a mini conference, but to have a hands-on and demo-heavy event to which participants can actively contribute by showing and discussing their research prototypes related to the topic. The workshop is structured into two parts.

In the first part, a particular focus is on demonstrations and discussions of existing systems and tools that have been developed, or are currently still under development, with the goal of supporting cross-device user interfaces. It is important to note that the workshop takes a much broader view on cross-device interfaces than is sometimes the case in the literature. The DUI workshop last held at EICS in London in 2013 is in topic related to this workshop. However, it set the focus on paper presentations by participants and only reserved little time for discussion, of which most was spent on defining DUI rather than discussing how to push forward in the field.

The second part is then a discussion moderated by the organisers to identify the key issues and important future directions with the goal of defining a common research agenda. This part of the workshop provides an important opportunity for new researchers interested in the topic to ask questions and for already active researchers to discuss a possible research 
agenda focusing on systematic approaches to the development of cross-device interfaces. In particular, the workshop's discussion addresses the following issues:

- experience reports regarding implementation of systems and tools as well as user studies: debating the issues around existing technologies and possible workarounds to mitigate remaining issues and address current limitations of existing systems and tools;

- the role of end-users and developers: many solutions have been specifically designed for developers, while others directly target end-users - there are tradeoffs and limitations with each that deserve attention;

- moving forward: discussing possibilities of forming an active working group; identifying topics, interested partners and funding opportunities for joint research projects; planning future workshops addressing related topics.

\section{Acknowledgements}

Michael Nebeling gratefully acknowledges the support provided by the Swiss National Science Foundation under grant P300P2_154571.

\section{REFERENCES}

1. Badam, S. K., and Elmqvist, N. PolyChrome: A Cross-Device Framework for Collaborative Web Visualization. In Proc. ITS (2014).

2. Chi, P. P., and Li, Y. Weave: Scripting Cross-Device Wearable Interaction. In Proc. CHI (2015).

3. Frosini, L., and Paternò, F. User Interface Distribution in Multi-Device and Multi-User Environments with Dynamically Migrating Engines. In Proc. EICS (2014).

4. Ghiani, G., Paternò, F., and Santoro, C. Push and Pull of Web User Interfaces in Multi-Device Environments. In Proc. AVI (2012).

5. Hamilton, P., and Wigdor, D. J. Conductor: Enabling and Understanding Cross-Device Interaction. In Proc. CHI (2014).

6. Hartmann, B., Beaudouin-Lafon, M., and Mackay, W. E. HydraScope: Creating Multi-Surface Meta-Applications Through View Synchronization and Input Multiplexing. In Proc. PerDis (2013).

7. Heikkinen, T., Goncalves, J., Kostakos, V., Elhart, I., and Ojala, T. Tandem Browsing Toolkit: Distributed Multi-Display Interfaces with Web Technologies. In Proc. PerDis (2014).

8. Houben, S., and Marquardt, N. WatchConnect: A Toolkit for Prototyping Smartwatch-Centric Cross-Device Applications. In Proc. CHI (2015).

9. Husmann, M., Nebeling, M., Pongelli, S., and Norrie, M. C. MultiMasher: Providing Architectural Support and Visual Tools for Multi-Device Mashups. In Proc. WISE (2014).
10. Melchior, J., Grolaux, D., Vanderdonckt, J., and Roy, P. V. A Toolkit for Peer-to-Peer Distributed User Interfaces: Concepts, Implementation, and Applications. In Proc. EICS (2009).

11. Melchior, J., Vanderdonckt, J., and Roy, P. V. A Model-Based Approach for Distributed User Interfaces. In Proc. EICS (2011).

12. Nebeling, M., Grossniklaus, M., Leone, S., and Norrie, M. C. XCML: Providing Context-Aware Language Extensions for the Specification of Multi-Device Web Applications. $W W W$ 15, 4 (2012).

13. Nebeling, M., Husmann, M., Zimmerli, C., Valente, G., and Norrie, M. C. XDSession: Integrated Development and Testing of Cross-Device Applications. In Proc. EICS (2015).

14. Nebeling, M., Mintsi, T., Husmann, M., and Norrie, M. C. Interactive Development of Cross-Device User Interfaces. In Proc. CHI (2014).

15. Nebeling, M., Teunissen, E., Husmann, M., and Norrie, M. C. XDKinect: Development Framework for Cross-Device Interaction using Kinect. In Proc. EICS (2014).

16. Nichols, J., and Myers, B. A. Creating a Lightweight User Interface Description Language: An Overview and Analysis of the Personal Universal Controller Project. TOCHI 16, 4 (2009).

17. Nichols, J., Myers, B. A., Higgins, M., Hughes, J., Harris, T. K., Rosenfeld, R., and Pignol, M. Generating Remote Control Interfaces for Complex Appliances. In Proc. UIST (2002).

18. Paternò, F., and Santoro, C. A Logical Framework for Multi-Device User Interfaces. In Proc. EICS (2012).

19. Paternò, F., Santoro, C., and Spano, L. MARIA: A Universal, Declarative, Multiple Abstraction-Level Language for Service-Oriented Applications in Ubiquitous Environments. TOCHI 16, 4 (2009).

20. Ruiz, J., Li, Y., and Lank, E. User-Defined Motion Gestures for Mobile Interaction. In Proc. CHI (2011).

21. Santosa, S., and Wigdor, D. A Field Study of Multi-Device Workflows in Distributed Workspaces. In Proc. UbiComp (2013).

22. Schmidt, D., Seifert, J., Rukzio, E., and Gellersen, H. A Cross-Device Interaction Style for Mobiles and Surfaces. In Proc. DIS (2012).

23. Seyed, T., Burns, C., Sousa, M. C., Maurer, F., and Tang, A. Eliciting Usable Gestures for Multi-Display Environments. In Proc. ITS (2012).

24. Yang, J., and Wigdor, D. Panelrama: Enabling Easy Specification of Cross-Device Web Applications. In Proc. CHI (2014). 